\title{
Control of Phosphate Excretion in Uremic Man
}

\author{
E. Slatopolsky, A. M. Robson, I. Elyan, and N. S. Bricker \\ From the Renal Division, Department of Internal Medicine, Washington \\ University School of Medicine, St. Louis, Missouri 63110
}

A B STRACT The present studies were performed in an effort to examine the characteristics of the control system governing phosphate excretion in uremic man. In a group of patients with glomerular filtration rates (GFR) ranging from normal to $2 \mathrm{ml} / \mathrm{min}$, it was found that the lower the GFR the lower the fraction of filtered phosphate reabsorbed (TRP). On a fixed phosphate intake, phosphate excretion rate was the same in patients with GFRs ranging from 60 to $3 \mathrm{ml} / \mathrm{min}$. When plasma phosphate concentrations were diminished to subnormal levels in hyperphosphatemic, hypocalcemic uremic patients, TRP values increased but did not return to normal. TRP failed to rise substantially when GFR, as well as plasma phosphate concentrations, were diminished. In patients with unilateral renal disease, TRP values were equal bilaterally, and values were substantially higher in the diseased kidneys than in patients with bilateral involvement. When plasma calcium concentrations were raised to normal for 2-3 wk in uremic patients in whom plasma phosphate concentrations had previously been lowered to subnormal levels, TRP values rose to an average value of $86 \%$. Values remained in the normal range when phosphate concentrations were allowed to increase while normocalcemia was maintained. The data are interpreted to indicate that in advancing renal disease, the changing patterns of phosphate excretion are mediated by a control system in which parathyroid hormone serves as a

This work was presented at the National Meeting of the American-Federation for Clinical Research, Atlantic City, N. J. An abstract has been published: Clin. Res. 1967. $15: 371$.

Dr. Ivan O. Elkan is a Research Associate at the John Cochran Veterans Administration Hospital.

Received for publication 4 March 1968 and in revised form 15 April 1968. major effector element. An increase in GFR per nephron, hyperphosphatemia, and intrinsic inability of the surviving nephrons to transport phosphate do not appear to be of primary importance in the progressive reduction in TRP.

\section{INTRODUCTION}

The maintenance of phosphate balance in health and on a normal diet requires that each nephron excrete only a small fraction of its filtered phosphate. In progressive renal disease, with a reduction in the number of functioning nephrons, similar rates of total daily phosphate excretion in the urine can only be maintained by an increased rate of phosphate excretion per nephron. We previously have presented evidence that in uremic dogs high rates of phosphate excretion per nephron are mediated in major part by increased parathyroid hormone activity (1). To date, however, efforts to distinguish between the role of parathyroid hormone, intrinsic disease of nephrons, and other factors in the phosphaturia observed in patients with chronic renal disease have not been uniformly successful. The present studies were undertaken therefore to examine the mechanisms which control the excretion of phosphate in uremic man.

\section{METHODS}

A total of 90 studies was performed in 24 patients with chronic progressive renal insufficiency. 13 patients had chronic glomerulonephritis, eight chronic pyelonephritis, and three polycystic renal disease.

All patients ingested a diet containing between 500 and $700 \mathrm{mg}$ of phosphate per day. Control observations consisting of three to four consecutive clearance periods were obtained in each patient to establish the relationship between phosphate clearance and glomerular filtration rate (GFR). In the uremic patients, the control studies thus were performed without modifying either the hyper- 
phosphatemia or hypocalcemia. A number of special studies also were performed in which the experimental conditions were altered in one or more ways.

Controlled phosphate intake. In four patients representing a wide range of GFRs, a special diet was employed to maintain phosphate intake at $650 \pm 50 \mathrm{mg} /$ day. Studies were performed after this regimen had been in effect for at least 5 days.

Divided function studies. Two patients with unilateral pyelonephritis underwent split-function studies, and urine was collected separately from the two kidneys by the use of ureteral catheters.

Reduction in plasma phosphate concentrations. Plasma phosphate levels were reduced to subnormal levels in five hyperphosphatemic uremic patients by the chronic administration of a low phosphate diet together with oral aluminum carbonate gel, 1 in a dose of $40-60 \mathrm{ml}$ four times per day. The initial phosphate concentrations in these patients ranged from 6.5 to $10.0 \mathrm{mg} / 100 \mathrm{ml}$ (mean $8.3 \mathrm{mg} / 100 \mathrm{ml}$ ) ; the final concentrations ranged from 1.4 to $3.6 \mathrm{mg} / 100 \mathrm{ml}$ (mean $2.4 \mathrm{mg} / 100 \mathrm{ml}$ ). The time required to reduce the plasma concentrations varied from 12 to 20 days.

Elevation of plasma calcium concentrations in the presence of low plasma phosphate concentrations. In six hypocalcemic patients in whom plasma phosphate levels had been reduced to subnormal levels in the manner described above, plasma calcium concentrations were raised to a range of $9.1-11.5 \mathrm{mg} / 100 \mathrm{ml}$ (mean $9.9 \mathrm{mg}$ / $100 \mathrm{ml}$ ) by the administration of $2-4 \mathrm{~g}$ of calcium gluconate four times daily and $200,000-300,000 \mathrm{U}$ of vitamin D daily. Plasma calcium concentrations were followed very closely to prevent the occurrence of progressive hypercalcemia. Studies were performed after the calcium concentration had been in a normal range for 2-3 wk, and results were compared with control studies.

Elevation of plasma phosphate concentrations to normal while normal plasma calcium concentrations zere maintained. In three hypophosphatemic, normocalcemic patients receiving vitamin $\mathrm{D}$ and calcium, plasma phosphate levels were allowed to increase to normal by diminishing the dose of aluminum carbonate gel. Calcium concentrations were maintained within a normal range. Studies were performed before and after the restoration of phosphate levels to the normal range.

Simultaneous reduction in GFR and plasma phosphate concentrations. Three patients were studied before and after an 8-10 hr period of hemodialysis with a twin-coil dialyzer. In each patient the initial (i.e. predialysis) glomerular filtration rate was below $4 \mathrm{ml} / \mathrm{min}$. GFR fell by an average of over $50 \%$ after dialysis, and plasma phosphate concentrations were reduced from a mean of $11.7 \mathrm{mg} / 100 \mathrm{ml}$ to $3.6 \mathrm{mg} / 100 \mathrm{ml}$.

Parathyroid hormone ( $P T H)$ infusion. In many studies, under most of the foregoing conditions, after three or more clearance periods had been obtained, parathyroid hormone was administered intravenously. $250 \mathrm{U}$ of $\mathrm{PTH}$ were infused over a 5-10 min interval after which $1 \mathrm{U} /$

\footnotetext{
${ }^{1}$ Basaljel, Wyeth Laboratories, Marietta, Pa.
}

min was delivered by continuous infusion throughout the remainder of the study. A period of 45-60 min was allowed for equilibration after the priming injection of $\mathrm{PTH}$, and then three or more additional clearance periods were obtained.

Glomerular filtration rate was measured by inulin clearance. Corrections were made for inuloid blank in blood and urine. Urine was collected through an indwelling bladder catheter, and at the end of each collection the bladder was washed with distilled water and air. Rigorous asepsis was used in performing the catheterizations; bacitracin-neomycin ointment was used as the catheter lubricant, and at the end of each study the bladder was irrigated with $200 \mathrm{ml}$ of $1 \%$ neomycin solution. A urine culture was obtained at the end of each study and repeated 3-4 days later. None of the patients developed a urinary tract infection. Individual clearance periods varied from 20 to $30 \mathrm{~min}$ in duration, and blood was collected at the midpoint of each clearance period through an indwelling venous catheter.

Inulin was measured according to the method of Roe, Epstein, and Goldstein (2), phosphate by the method of Gomori (3), and calcium with a Perkin-Elmer atomic absorption spectrophotometer. Additional details of the experimental procedures employed have been described (4). TRP, the conventional expression for the apparent fractional reabsorption of phosphate, has been employed in describing the experimental results: $\left(T R P=\left(1-\mathrm{C}_{\mathbf{P O}_{4}} /\right.\right.$ GFR) $\times 100$, where $\mathrm{C}_{\mathrm{PO}_{4}}=$ phosphate clearance). The fraction of filtered phosphate excreted $\left(\mathrm{C}_{\mathrm{PO}_{4}} / \mathrm{GFR} \times 100\right)$ also has been used. The validity of both of these expressions requires a phosphate excretory system which does not include a secretory component; but if secretion does occur, the basic observations and their biologic meaning would not be modified. No corrections for Donnan equilibrium or protein binding of phosphate were employed in the calculations.

\section{RESULTS}

Fig. 1 illustrates the relationship between TRP and glomerular filtration rate in 24 patients with bilateral renal disease varying in severity from moderately advanced to far advanced. Data from six normal individuals are included for comparison. A hyperbolic function is defined by the data such that the lower the steady-state GFR, the lower the value for TRP. With very low glomerular filtration rates, as little as $10-20 \%$ of the filtered phosphate is reabsorbed. When the fraction of filtered phosphate excreted rather than TRP is plotted on the ordinate, there is approximately a twofold increase in $\mathrm{C}_{\mathrm{PO}_{4}} / \mathrm{GFR}$ for every $50 \%$ decrease in GFR. The over-all pattern described by the data in Fig. 1 is reminiscent of the results previously reported by Goldman and Bassett (5). The patterns of phosphate excretion in the indi- 


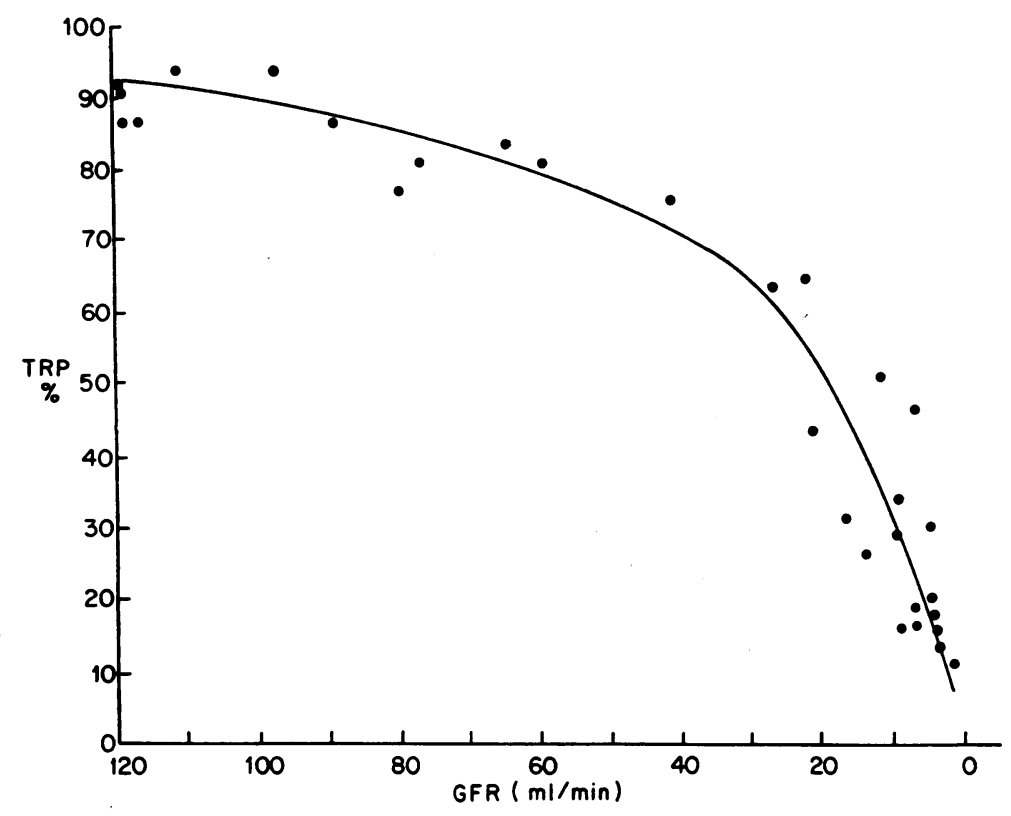

Figure 1 Comparison of tubular reabsorption of phosphate $(T R P)$ with glomerular filtration rates $(G F R)$ for a group of 24 patients with chronic progressive bilateral renal disease and six normal individuals. Each point represents a mean value of at least three consecutive clearance periods. Data are not corrected for surface area, but a separate plot expressing $\mathrm{GFR} / 1.73 \mathrm{~m}^{2}$ of body surface area is essentially superimposable. vidual kidneys of two patients with unilateral pyelonephritis are shown in Fig. 2. In one patient, values for GFR in the two kidneys were 37 and $64 \mathrm{ml} / \mathrm{min}$ respectively; in the second patient, the values were 11 and $70 \mathrm{ml} / \mathrm{min}$. In each patient, despite the disparity in GFR between the two kidneys, values for TRP were equal bilaterally.

The results of the studies performed on the four patients maintained on a carefully controlled phosphate intake $(650 \pm 50 \mathrm{mg} /$ day $)$ are shown in Fig. 3. Although GFR ranged from 60 to $3 \mathrm{ml} /$

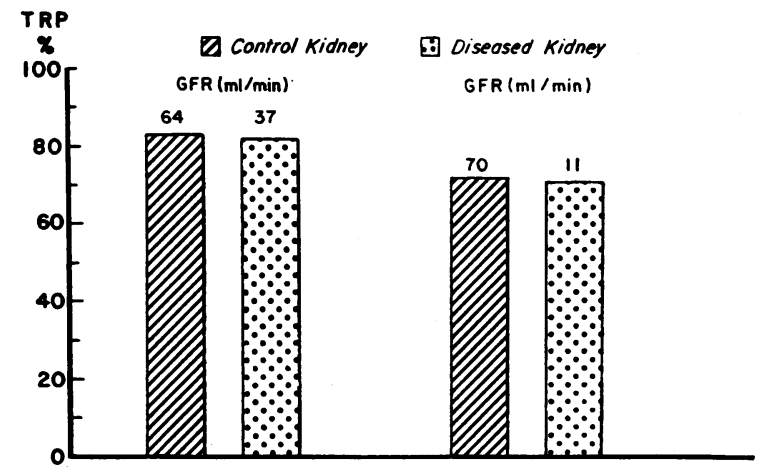

Figure 2 Simultaneous measurements of TRP in the individual kidneys of two patients with unilateral chronic pyelonephritis. Urine was collected from the individual kidneys via ureteral catheters, and appropriate precautions were taken to preclude significant leak about the catheters. These studies were performed for other purposes, and the opportunity to measure TRP thus became available fortuitously. min, the phosphate excretion rates were virtually the same in all patients. The values for TRP in these patients are included in Fig. 3. As in Fig. 1, the lower the GFR, the lower the TRP. Thus, the ability to maintain constant phosphate excretion rates with decreasing levels of GFR (and presumably a decreasing population of functioning nephrons) was facilitated by the progressive reduction in the fraction of filtered phosphate reabsorbed. In the two patients with the lowest glomerular filtration rates ( 8 and $3 \mathrm{ml} / \mathrm{min}$ ), plasma phosphate concentrations were elevated, and the increase in filtered phosphate also may be assumed to have contributed to the sustained phosphaturia (per nephron).

In order to study the importance of hyperphosphatemia in the progressive reduction of TRP that occurs with advancing nephron destruction, studies were performed in five patients before and after plasma phosphate concentrations were reduced from high to subnormal levels by the administration of aluminum carbonate gel. These data are shown in Table I. The plasma phosphate concentrations averaged $8.3 \mathrm{mg} / 100 \mathrm{ml}$ in the control studies and $2.4 \mathrm{mg} / 100 \mathrm{ml}$ in the follow-up studies. Plasma calcium levels increased from an average of 7.6 to $8.3 \mathrm{mg} / 100 \mathrm{ml}$; however, in no patient did calcium concentrations increase to a normal range. TRP averaged $17 \%$ (range 12.6$21.4 \%$ ) in the hyperphosphatemic state; in the 


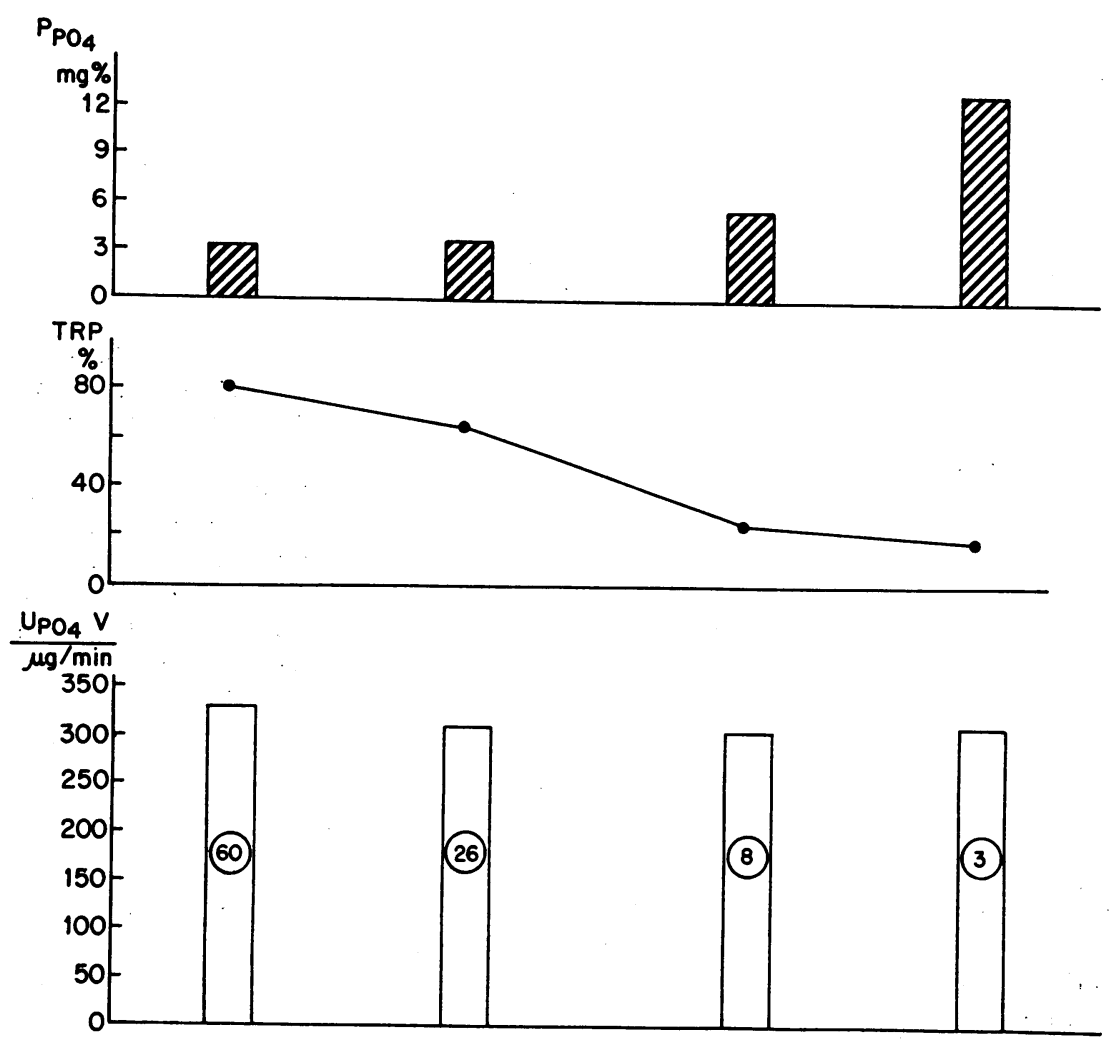

FIGURE 3 Patterns of phosphate excretion $\left(U_{P O_{4}} V\right)$ in four patients with a range of GFR from 60 to $3 \mathrm{ml} / \mathrm{min}$ while they were on a fixed phosphate intake $(650 \pm 50$ $\mathrm{mg} /$ day). The values for each patient represent the mean of at least three consecutive clearance periods. $P_{P_{4}}$, plasma phosphate concentration.

presence of hypophosphatemia TRP increased to a mean value of $41.9 \%$ (range $32.8-55.8 \%$ ). Net phosphate reabsorption fell slightly from 70 to 62 $\mu \mathrm{g} / \mathrm{min}$, and phosphate excretion fell from a mean of 350 to $83 \mu \mathrm{g} / \mathrm{min}$. (The filtered load of phosphate fell from a mean of 420 to $145 \mu \mathrm{g} / \mathrm{min}$.)

The effects of parathyroid hormone administration were examined before and after the reversal of hyperphosphatemia. The data are included in Table I. In the control (i.e. hyperphosphatemic) studies, PTH had essentially no effect on TRP. After reduction of phosphate concentrations, the administration of $\mathrm{PTH}$ was associated with a consistent, albeit very small, decrease in TRP; (the mean value fell from 41.9 to $37.8 \%$ ). Net phosphate reabsorption also decreased modestly (from a mean of 62 to $49 \mu \mathrm{g} / \mathrm{min}$ ).

The results of decreasing GFR, as well as plasma phosphate concentrations, by hemodialysis are shown in Table II. In each of the three patients studied, GFR fell by more than $45 \%$, and plasma phosphate concentrations fell by an average of $69 \%$ (initial mean value $11.7 \mathrm{mg} / 100 \mathrm{ml}$; postdialysis value $3.6 \mathrm{mg} / 100 \mathrm{ml}$ ). The plasma calcium concentrations increased only slightly from 7.7 to 8.5 $\mathrm{mg} / 100 \mathrm{ml}$. Despite the reduction in both determinants of the filtered load of phosphate (GFR and plasma phosphate concentrations), TRP rose only from a mean of 14.3 to $26.7 \%$.

Table III depicts the results of the studies performed in the six patients in whom hypophosphatemia was induced with aluminum carbonate gel, and then plasma calcium concentrations were elevated and maintained between 9 and $11.5 \mathrm{mg} / 100$ $\mathrm{ml}$ for 2-3 wk. ${ }^{2}$ The increase in plasma calcium concentrations was associated with a striking rise in values for TRP. In five of the six patients, TRP values exceeded $85 \%$; and in one patient the value

2 The TRP values were estimated every 2-3 days by using creatinine clearances, and values were found to increase slowly, not reaching the normal range for approximately 2 wk. 
TABLE I

Effects of Decreasing Plasma Phosphate Concentrations of $\mathrm{PO}_{4}$ Excretion ${ }^{*}$

\begin{tabular}{|c|c|c|c|c|c|c|c|c|c|}
\hline Patient & Condition & GFR & PrO4 & $\mathrm{Pc}_{\mathrm{a}}$ & TRP & CPO4 & FLPO4 & UPO4V & $\mathrm{T}_{\mathrm{PO}}$ \\
\hline 1 & $\begin{array}{l}\text { Control } \\
\text { PTH }\end{array}$ & $\begin{array}{c}m l / m i n \\
2.62 \\
3.22\end{array}$ & $\begin{array}{c}m g / 100 m l \\
10.0 \\
9.9\end{array}$ & $\begin{array}{c}m g / 100 m l \\
8.40 \\
8.42\end{array}$ & $\begin{array}{c}\% \\
12.6 \\
14.6\end{array}$ & $\begin{array}{c}m l / \min \\
2.29 \\
2.75\end{array}$ & $\begin{array}{c}\mu g / \min \\
262 \\
319\end{array}$ & $\begin{array}{c}\mu g / \min \\
229 \\
272\end{array}$ & $\begin{array}{c}\mu g / \min \\
33 \\
47\end{array}$ \\
\hline & $\begin{array}{l}\mathrm{PO}_{4} \text { depletion } \\
\text { PTH }\end{array}$ & $\begin{array}{l}3.02 \\
3.38\end{array}$ & $\begin{array}{l}1.4 \\
1.4\end{array}$ & $\begin{array}{l}9.2 \\
9.2\end{array}$ & $\begin{array}{l}32.8 \\
30.8\end{array}$ & $\begin{array}{l}2.03 \\
2.39\end{array}$ & $\begin{array}{l}42 \\
47\end{array}$ & $\begin{array}{l}29 \\
33\end{array}$ & $\begin{array}{l}13 \\
14\end{array}$ \\
\hline 2 & $\begin{array}{l}\text { Control } \\
\text { PTH }\end{array}$ & $\begin{array}{l}4.34 \\
4.65\end{array}$ & $\begin{array}{l}7.83 \\
7.50\end{array}$ & $\begin{array}{l}7.55 \\
7.33\end{array}$ & $\begin{array}{l}21.4 \\
14.6\end{array}$ & $\begin{array}{l}3.41 \\
3.97\end{array}$ & $\begin{array}{l}340 \\
349\end{array}$ & $\begin{array}{l}266 \\
299\end{array}$ & $\begin{array}{l}74 \\
50\end{array}$ \\
\hline & $\begin{array}{l}\mathrm{PO}_{4} \text { depletion } \\
\mathrm{PTH}\end{array}$ & $\begin{array}{l}6.66 \\
5.32\end{array}$ & $\begin{array}{l}2.60 \\
2.36\end{array}$ & $\begin{array}{l}8.50 \\
8.45\end{array}$ & $\begin{array}{l}55.8 \\
47.6\end{array}$ & $\begin{array}{l}2.94 \\
2.77\end{array}$ & $\begin{array}{l}173 \\
125\end{array}$ & $\begin{array}{l}76 \\
66\end{array}$ & $\begin{array}{l}97 \\
59\end{array}$ \\
\hline 3 & $\begin{array}{l}\text { Control } \\
\text { PTH }\end{array}$ & $\begin{array}{l}4.21 \\
3.91\end{array}$ & $\begin{array}{l}8.6 \\
8.4\end{array}$ & $\begin{array}{l}6.87 \\
6.97\end{array}$ & $\begin{array}{l}18.5 \\
14.1\end{array}$ & $\begin{array}{l}3.43 \\
3.36\end{array}$ & $\begin{array}{l}362 \\
328\end{array}$ & $\begin{array}{l}296 \\
281\end{array}$ & $\begin{array}{l}66 \\
47\end{array}$ \\
\hline & $\begin{array}{l}\mathrm{PO}_{4} \text { depletion } \\
\mathrm{PTH}\end{array}$ & $\begin{array}{l}4.25 \\
4.22\end{array}$ & $\begin{array}{l}2.73 \\
3.07\end{array}$ & $\begin{array}{l}7.07 \\
7.57\end{array}$ & $\begin{array}{l}39.1 \\
38.2\end{array}$ & $\begin{array}{l}2.59 \\
2.61\end{array}$ & $\begin{array}{l}116 \\
130\end{array}$ & $\begin{array}{l}72 \\
83\end{array}$ & $\begin{array}{l}44 \\
47\end{array}$ \\
\hline 4 & $\begin{array}{l}\text { Control } \\
\text { PTH }\end{array}$ & $\begin{array}{l}12.0 \\
13.7\end{array}$ & $\begin{array}{l}6.5 \\
6.5\end{array}$ & $\begin{array}{l}7.05 \\
7.15\end{array}$ & $\begin{array}{l}15.0 \\
25.6\end{array}$ & $\begin{array}{l}10.2 \\
10.2\end{array}$ & $\begin{array}{l}780 \\
891\end{array}$ & $\begin{array}{l}663 \\
660\end{array}$ & $\begin{array}{l}117 \\
231\end{array}$ \\
\hline & $\begin{array}{l}\mathrm{PO}_{4} \text { depletion } \\
\mathrm{PTH}\end{array}$ & $\begin{array}{l}9.13 \\
9.34\end{array}$ & $\begin{array}{l}1.7 \\
1.6\end{array}$ & $\begin{array}{l}8.2 \\
8.2\end{array}$ & $\begin{array}{l}43.8 \\
40.8\end{array}$ & $\begin{array}{l}5.12 \\
5.54\end{array}$ & $\begin{array}{l}155 \\
149\end{array}$ & $\begin{array}{l}86 \\
89\end{array}$ & $\begin{array}{l}69 \\
60\end{array}$ \\
\hline 5 & $\begin{array}{l}\text { Control } \\
\text { PTH }\end{array}$ & $\begin{array}{l}4.09 \\
4.50\end{array}$ & $\begin{array}{l}8.7 \\
7.9\end{array}$ & $\begin{array}{l}8.0 \\
8.1\end{array}$ & $\begin{array}{l}17.8 \\
18.0\end{array}$ & $\begin{array}{l}3.36 \\
3.69\end{array}$ & $\begin{array}{l}356 \\
356\end{array}$ & $\begin{array}{l}295 \\
292\end{array}$ & $\begin{array}{l}61 \\
64\end{array}$ \\
\hline & $\begin{array}{l}\mathrm{PO}_{4} \text { depletion } \\
\mathrm{PTH}\end{array}$ & $\begin{array}{l}6.63 \\
5.53\end{array}$ & $\begin{array}{l}3.6 \\
3.4\end{array}$ & $\begin{array}{l}8.4 \\
8.4\end{array}$ & $\begin{array}{l}38.2 \\
31.8\end{array}$ & $\begin{array}{l}4.09 \\
3.61\end{array}$ & $\begin{array}{l}237 \\
188\end{array}$ & $\begin{array}{l}150 \\
124\end{array}$ & $\begin{array}{l}87 \\
64\end{array}$ \\
\hline Mean & $\begin{array}{l}\text { Control } \\
\text { PTH }\end{array}$ & $\begin{array}{l}5.45 \\
6.00\end{array}$ & $\begin{array}{l}8.33 \\
8.04\end{array}$ & $\begin{array}{l}7.57 \\
7.59\end{array}$ & $\begin{array}{l}17.0 \\
17.4\end{array}$ & $\begin{array}{l}4.54 \\
4.79\end{array}$ & $\begin{array}{l}420 \\
449\end{array}$ & $\begin{array}{l}350 \\
361\end{array}$ & $\begin{array}{l}70 \\
88\end{array}$ \\
\hline & $\begin{array}{l}\mathrm{PO}_{4} \text { depletion } \\
\text { PTH }\end{array}$ & $\begin{array}{l}5.94 \\
5.56\end{array}$ & $\begin{array}{l}2.40 \\
2.37\end{array}$ & $\begin{array}{l}8.27 \\
8.36\end{array}$ & $\begin{array}{l}41.9 \\
37.8\end{array}$ & $\begin{array}{l}3.35 \\
3.77\end{array}$ & $\begin{array}{l}145 \\
128\end{array}$ & $\begin{array}{l}83 \\
79\end{array}$ & $\begin{array}{l}62 \\
49\end{array}$ \\
\hline
\end{tabular}

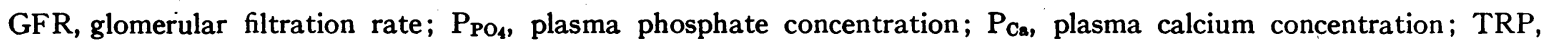
tubular reabsorption of phosphate; $\mathrm{C}_{\mathrm{PO}_{4}}$, clearance of phosphate; FL $\mathrm{PO}_{4}$, filtered load of phosphate; $\mathrm{U}_{\mathrm{PO}_{4} \mathrm{~V}}$, phosphate excreted; $\mathrm{T}_{\text {PO4}}$, phosphate reabsorbed; PTH, parathyroid hormone. No Donnan correction was applied in calculating the filtered load of phosphate.

* All results are the average of three or more clearance periods in this and succeeding tables.

TABLE II

Effects of Decreasing GFR and Plasma Phosphate Concentrations on POA Excretion

\begin{tabular}{|c|c|c|c|c|c|c|c|c|c|}
\hline Patient & Condition & GFR & PPO4 & $\mathrm{Pc}_{\mathrm{a}}$ & TRP & $\mathrm{CPO}_{4}$ & FLPO4 & $\mathrm{UPO}_{4} \mathrm{~V}$ & $T_{P O A}$ \\
\hline 1 & $\begin{array}{l}\text { Predialysis } \\
\text { Postdialysis }\end{array}$ & $\begin{array}{c}m l / m i n \\
3.25 \\
1.21\end{array}$ & $\begin{array}{c}m g / 100 m l \\
10.2 \\
3.3\end{array}$ & $\begin{array}{c}m g / m l \\
7.9 \\
8.5\end{array}$ & $\begin{array}{c}\% \\
8 \\
14.9\end{array}$ & $\begin{array}{c}m l / \min \\
2.99 \\
1.03\end{array}$ & $\begin{array}{c}\mu g / \min \\
332 \\
40\end{array}$ & $\begin{array}{c}\mu g / \min \\
305 \\
34\end{array}$ & $\begin{array}{c}\mu g / \min \\
27 \\
6\end{array}$ \\
\hline 2 & $\begin{array}{l}\text { Predialysis } \\
\text { Postdialysis }\end{array}$ & $\begin{array}{l}3.14 \\
1.53\end{array}$ & $\begin{array}{r}11.0 \\
2.7\end{array}$ & $\begin{array}{l}8.0 \\
8.4\end{array}$ & $\begin{array}{l}13.4 \\
37.9\end{array}$ & $\begin{array}{l}2.72 \\
0.95\end{array}$ & $\begin{array}{r}345 \\
41\end{array}$ & $\begin{array}{r}299 \\
26\end{array}$ & $\begin{array}{l}46 \\
15\end{array}$ \\
\hline 3 & $\begin{array}{l}\text { Predialysis } \\
\text { Postdialysis }\end{array}$ & $\begin{array}{l}3.16 \\
1.64\end{array}$ & $\begin{array}{r}14.0 \\
4.7\end{array}$ & $\begin{array}{l}7.25 \\
8.60\end{array}$ & $\begin{array}{l}21.5 \\
27.4\end{array}$ & $\begin{array}{l}2.48 \\
1.19\end{array}$ & $\begin{array}{r}442 \\
77\end{array}$ & $\begin{array}{r}322 \\
57\end{array}$ & $\begin{array}{r}120 \\
20\end{array}$ \\
\hline Mean & $\begin{array}{l}\text { Predialysis } \\
\text { Postdialysis }\end{array}$ & $\begin{array}{l}3.18 \\
1.46\end{array}$ & $\begin{array}{l}11.7 \\
3.57\end{array}$ & $\begin{array}{l}7.72 \\
8.5\end{array}$ & $\begin{array}{l}14.3 \\
26.7\end{array}$ & $\begin{array}{l}2.73 \\
1.06\end{array}$ & $\begin{array}{r}373 \\
53\end{array}$ & $\begin{array}{r}309 \\
39\end{array}$ & $\begin{array}{l}64 \\
14\end{array}$ \\
\hline
\end{tabular}

See Table I for explanation of abbreviations. 
was $97.6 \%$. For the group the mean value rose from 16.1 to $86 \%$. The filtered load of phosphate was reduced from an initial mean value of 413 to $114 \mu \mathrm{g} / \mathrm{min}$. Yet, in contrast to the results observed with reduction in plasma phosphate levels alone or with reduction in both GFR and plasma phosphate levels, net tubular reabsorption of phosphate rose from 68 to $102 \mu \mathrm{g} / \mathrm{min}$, and phosphate excretion rates fell from an average control rate of 346 to $12 \mu \mathrm{g} / \mathrm{min}$. The effects of PTH admin- istration in the normocalcemic hypophosphatemic state are shown in Table III. Whereas administration of $\mathrm{PTH}$ had virtually no effect in patients with hypocalcemia and hyperphosphatemia (Table I) and had only a very slight effect in patients with hypocalcemia and hypophosphatemia (Table II), a somewhat more marked influence was observed in the normocalcemic hypophosphatemic patients. Thus, the average value for TRP decreased from a mean of $86 \%$ before the adminis-

TABLE III

Effects of Sustained Elevation of Plasma Calcium Concentrations on $\mathrm{PO}_{4}$ Excretion in Hypophosphatemic Patients

\begin{tabular}{|c|c|c|c|c|c|c|c|c|c|}
\hline Patient & Condition & GFR & $\mathrm{PPO}_{4}$ & $\mathrm{Pc}$ & TRP & $\mathrm{CPO}_{4}$ & FLPO4 & $\mathrm{UPO}_{4} \mathrm{~V}$ & TPO4 \\
\hline \multirow[t]{3}{*}{1} & Control & $\begin{array}{c}\mathrm{ml} / \mathrm{min} \\
12.0\end{array}$ & $\begin{array}{c}m g / 100 \mathrm{ml} \\
6.5\end{array}$ & $\begin{array}{c}m g / 100 m l \\
7.05\end{array}$ & 15 & $\begin{array}{l}\mathrm{ml} / \mathrm{min} \\
10.2\end{array}$ & $\begin{array}{c}\mu g / \min \\
780\end{array}$ & $\begin{array}{c}\mu g / \min \\
663\end{array}$ & $\begin{array}{c}\mu g / \min \\
117\end{array}$ \\
\hline & $\begin{array}{l}\mathrm{PO}_{4} \text { depletion, } \\
\text { vitamin } \mathrm{D}+\mathrm{Ca}\end{array}$ & 8.27 & 2.7 & 9.00 & 97.6 & 0.2 & 223 & 5 & 218 \\
\hline & PTH & 10.4 & 2.3 & 9.00 & 90.0 & 1.04 & 239 & 26 & 213 \\
\hline \multirow[t]{3}{*}{2} & Control & 4.21 & 8.6 & 6.87 & 18.5 & 3.43 & 362 & 296 & 66 \\
\hline & $\begin{array}{l}\mathrm{PO}_{4} \text { depletion, } \\
\text { vitamin } \mathrm{D}+\mathrm{Ca}\end{array}$ & 4.74 & 1.23 & 9.33 & 85.7 & 0.68 & 58 & 8 & 50 \\
\hline & PTH & 4.08 & 1.33 & 9.62 & 66.4 & 1.37 & 54 & 18 & 36 \\
\hline \multirow[t]{3}{*}{3} & Control & 4.34 & 7.83 & 7.55 & 21.4 & 3.41 & 340 & 267 & 73 \\
\hline & $\begin{array}{l}\mathrm{PO}_{4} \text { depletion, } \\
\quad \text { vitamin } \mathrm{D}+\mathrm{Ca}\end{array}$ & 6.32 & 3.00 & 10.2 & 86.9 & 0.83 & 190 & 25 & 165 \\
\hline & PTH & 7.95 & 2.77 & 10.2 & 70.4 & 2.35 & 220 & 64 & 156 \\
\hline \multirow[t]{2}{*}{4} & $\begin{array}{l}\text { Control } \\
\mathrm{PO}_{4} \text { depletion, } \\
\quad \text { vitamin } \mathrm{D}+\mathrm{Ca}\end{array}$ & $\begin{array}{l}2.62 \\
3.31\end{array}$ & $\begin{array}{r}10.0 \\
1.6\end{array}$ & $\begin{array}{r}8.4 \\
11.5\end{array}$ & $\begin{array}{l}12.6 \\
70.7\end{array}$ & $\begin{array}{l}2.29 \\
0.97\end{array}$ & $\begin{array}{r}262 \\
53\end{array}$ & $\begin{array}{r}229 \\
15\end{array}$ & $\begin{array}{l}33 \\
38\end{array}$ \\
\hline & PTH & 3.33 & 1.5 & 11.3 & 26.4 & 2.45 & 50 & 37 & 13 \\
\hline \multirow[t]{3}{*}{5} & Control & 4.38 & 8.67 & 7.93 & 14.2 & 3.76 & 380 & 324 & 56 \\
\hline & $\begin{array}{l}\mathrm{PO}_{4} \text { depletion, } \\
\quad \text { vitamin } \mathrm{D}+\mathrm{Ca}\end{array}$ & 3.21 & 1.4 & 9.13 & 87.2 & 0.41 & 45 & 6 & 39 \\
\hline & PTH & 3.76 & 1.67 & 9.33 & 71.8 & 1.06 & 63 & 18 & 45 \\
\hline \multirow[t]{3}{*}{6} & Control & 4.09 & 8.7 & 8.0 & 17.8 & 3.36 & 356 & 295 & 61 \\
\hline & $\begin{array}{l}\mathrm{PO}_{4} \text { depletion, } \\
\quad \text { vitamin } \mathrm{D}+\mathrm{Ca}\end{array}$ & 5.99 & 1.9 & 10.3 & 88.1 & 0.71 & 114 & 13 & 101 \\
\hline & PTH & 6.33 & 1.9 & 10.3 & 77.6 & 1.42 & 120 & 27 & 93 \\
\hline \multirow[t]{3}{*}{ Mean } & Control & 5.27 & 8.38 & 7.63 & 16.1 & 4.41 & 413 & 346 & 68 \\
\hline & $\begin{array}{l}\mathrm{PO}_{4} \text { depletion, } \\
\quad \text { vitamin } \mathrm{D}+\mathrm{Ca}\end{array}$ & 5.31 & 1.97 & 9.91 & 86.0 & 0.63 & 114 & 12 & 102 \\
\hline & PTH & 5.98 & 1.91 & 9.96 & 67.1 & 1.62 & 124 & 32 & 92 \\
\hline
\end{tabular}

See Table I for explanation of abbreviations. 
TABLE IV

Effects of Elevation of Plasma Phosphate in Patients with Sustained Normocalcemia

\begin{tabular}{|c|c|c|c|c|c|c|c|c|c|}
\hline Patient & Condition & GFR & Pro4 & $\mathrm{Pc}_{\mathrm{a}}$ & TRP & $\mathrm{CPO}_{\mathrm{PO}}$ & FLPO & $\mathrm{UPO}_{4} \mathrm{~V}$ & TPO4 \\
\hline \multirow[t]{4}{*}{1} & Control & $\begin{array}{c}\mathrm{ml} / \mathrm{min} \\
4.21\end{array}$ & $\begin{array}{c}m g / 100 \mathrm{ml} \\
8.60\end{array}$ & $\begin{array}{c}m g / 100 \mathrm{ml} \\
6.87\end{array}$ & $\begin{array}{c}\% \\
18.5\end{array}$ & $\begin{array}{l}m l / \min \\
3.43\end{array}$ & $\begin{array}{c}\mu g / \min \\
362\end{array}$ & $\begin{array}{c}\mu g / \min \\
296\end{array}$ & $\begin{array}{c}\mu g / \min \\
66\end{array}$ \\
\hline & $\begin{array}{l}\mathrm{PO}_{4} \text { depletion, } \\
\text { vitamin } \mathrm{D}+\mathrm{Ca}\end{array}$ & 4.74 & 1.23 & 9.33 & 85.7 & 0.68 & 58 & 8 & 50 \\
\hline & $\begin{array}{l}\text { Normophosphatemia, } \\
\text { vitamin } D+C a\end{array}$ & 3.62 & 4.2 & 10.2 & 78.2 & 0.79 & 152 & 33 & 119 \\
\hline & PTH & 3.66 & 4.2 & 10.3 & 51.6 & 1.77 & 154 & 75 & 79 \\
\hline \multirow[t]{4}{*}{2} & Control & 4.34 & 7.83 & 7.55 & 21.4 & 3.41 & 340 & 267 & 73 \\
\hline & $\begin{array}{l}\mathrm{PO}_{4} \text { depletion, } \\
\quad \text { vitamin } \mathrm{D}+\mathrm{Ca}\end{array}$ & 6.32 & 3.00 & 10.2 & 86.9 & 0.83 & 190 & 25 & 165 \\
\hline & $\begin{array}{l}\text { Normophosphatemia, } \\
\text { vitamin } D+C a\end{array}$ & 5.49 & 5.0 & 9.9 & 85.1 & 0.82 & 275 & 41 & 234 \\
\hline & РTH & 6.55 & 4.57 & 9.8 & 63.0 & 2.42 & 299 & 111 & 188 \\
\hline \multirow[t]{4}{*}{3} & Control & 12.0 & 6.5 & 7.05 & 15.0 & 10.2 & 780 & 663 & 117 \\
\hline & $\begin{array}{l}\mathrm{PO}_{4} \text { depletion, } \\
\quad \text { vitamin } \mathrm{D}+\mathrm{Ca}\end{array}$ & 8.27 & 2.7 & 9.0 & 97.6 & 0.2 & 223 & 5 & 218 \\
\hline & $\begin{array}{l}\text { Normophosphatemia, } \\
\text { vitamin } D+C a\end{array}$ & 8.05 & 3.8 & 9.8 & 95.0 & 0.4 & 306 & 15 & 291 \\
\hline & PTH & 8.51 & 3.1 & 9.9 & 84.3 & 1.34 & 264 & 41 & 223 \\
\hline \multirow[t]{4}{*}{ Mean } & Control & 6.85 & 7.71 & 7.16 & 18.3 & 5.68 & 494 & 409 & 85 \\
\hline & $\begin{array}{l}\mathrm{PO}_{4} \text { depletion, } \\
\quad \text { vitamin } \mathrm{D}+\mathrm{Ca}\end{array}$ & 6.44 & 2.31 & 9.51 & 90.1 & 0.57 & 157 & 13 & 144 \\
\hline & Normophosphatemia & 5.72 & 4.33 & 9.97 & 86.1 & 0.67 & 244 & 30 & 214 \\
\hline & PTH & 6.24 & 3.96 & 10.0 & 66.3 & 1.84 & 239 & 76 & 163 \\
\hline
\end{tabular}

See Table I for explanation of abbreviations.

tration of PTH to $67.1 \%$. Net tubular reabsorption of phosphate also diminished modestly in association with PTH administration, but values did not return to the level observed before calcium concentrations were restored to normal.

A final set of experiments was performed to determine the effects of elevation of plasma phosphate concentrations from a low to a normal range in patients in whom plasma calcium concentrations were maintained within a normal range. These studies are shown in Table IV. The TRP values remained closely comparable to those observed in the hypophosphatemic state (i.e. 78.2, 85.1, and $95 \%$ vs. $85.7,86.9$, and $97.6 \%$ ) and were strikingly greater than the average control value of $18.3 \%$. Net phosphate reabsorption increased between the control study (hyperphosphatemia and hypocalcemia) and the final study (normophosphatemia and normocalcemia) by $250 \%$ despite a decrease in the filtered load of phosphate of over $50 \%$, and phosphate excretion fell concomitantly from 409 to $30 \mu \mathrm{g} / \mathrm{min}$. When PTH was administered in the presence of normophosphatemia and normocalcemia, TRP fell modestly in each patient, and phosphate reabsorption decreased from an average of 214 to $163 \mu \mathrm{g} / \mathrm{min}$.

In Table $\mathrm{V}$, the results of the sequential studies described above are shown in a single patient. In the control studies with hyperphosphatemia and hypocalcemia TRP averaged $21.4 \%$. With reduction in plasma phosphate concentrations but persistence of hypocalcemia, the TRP rose to $55.9 \%$. With elevation of calcium concentrations to normal and persisting hypophosphatemia, TRP 
TABLE V

Sequential Studies on a Single Patient

\begin{tabular}{|c|c|c|c|c|c|c|c|c|}
\hline Condition & $\mathrm{C}_{\text {In }}$ & $\mathrm{PpO}_{4}$ & $\mathrm{P}_{\mathrm{Ca}}$ & TRP & CPO4 & FLPO4 & $\mathrm{UPO}_{4} \mathrm{~V}$ & $\mathrm{TPO}_{4}$ \\
\hline & $\mathrm{ml} / \mathrm{min}$ & $m g / 100 \mathrm{ml}$ & $\mathrm{mg} / 100 \mathrm{ml}$ & $\%$ & $\mathrm{ml} / \mathrm{min}$ & $\mu g / \min$ & $\mu g / \min$ & $\mu_{\mathscr{G}} / \min$ \\
\hline Control & 4.34 & 7.83 & 7.55 & 21.4 & 3.41 & 340 & 266 & 74 \\
\hline PTH & 4.65 & 7.5 & 7.33 & 14.6 & 3.97 & 349 & 299 & 50 \\
\hline \multicolumn{9}{|c|}{$\mathrm{PO}_{4}$ depletion } \\
\hline Control & 6.66 & 2.60 & 8.5 & 55.9 & 2.94 & 173 & 76 & 97 \\
\hline PTH & 5.32 & 2.36 & 8.45 & 47.9 & 2.77 & 126 & 66 & 60 \\
\hline \multicolumn{9}{|c|}{$\mathrm{PO}_{4}$ depletion + vitamin $\mathrm{D}+\mathrm{Ca}$} \\
\hline Control & 6.32 & 3.0 & 10.2 & 86.9 & 0.83 & 190 & 25 & 165 \\
\hline PTH & 7.95 & 2.77 & 10.2 & 70.4 & 2.35 & 220 & 64 & 156 \\
\hline \multicolumn{9}{|c|}{ Normophosphatemia + vitamin $\mathrm{D}+\mathrm{Ca}$} \\
\hline Control & 5.49 & 5.0 & 9.9 & 85.1 & 0.82 & 275 & 41 & 234 \\
\hline PTH & 6.55 & 4.57 & 9.8 & 63.1 & 2.42 & 299 & 111 & 188 \\
\hline
\end{tabular}

$\mathrm{C}_{\mathrm{In}}$, inulin clearance. See Table I for explanation of other abbreviations.

increased to $86.9 \%$. Then with calcium concentrations maintained within a normal range and elevation of plasma phosphate concentrations to normal, TRP remained at $85 \%$. Net phosphate reabsorption averaged $74 \mu \mathrm{g} / \mathrm{min}$ in the control study; it increased to $97 \mu \mathrm{g} / \mathrm{min}$ with reduction of plasma phosphate concentrations to subnormal levels; it increased further to $165 \mu \mathrm{g} / \mathrm{min}$ with the induction of normocalcemia; and a further increase to 234 $\mu \mathrm{g} / \mathrm{min}$ was observed when plasma phosphate concentrations were increased to normal while normocalcemia was maintained. PTH had essentially no effect in the control study. It produced a slight change in the hypophosphatemic, hypocalcemic state; the change was more marked in the presence of a normal calcium concentration.

\section{DISCUSSION}

We have attempted in the present studies to examine the mechanisms responsible for the regulated increase in phosphate excretion rate per nephron in man with advancing renal disease and a progressive reduction in the nephron population. Theoretically, the phosphaturic response could result from an impairment in the capacity of the urine-forming nephrons to reabsorb phosphate due to the effects of the underlying renal lesion; it could result from an increase in the filtered load of phosphate produced by an increase in GFR per nephron or by hyperphosphatemia; or there could be a sensitive control mechanism which is con- cerned either directly or indirectly with the maintenance of phosphate homeostasis.

The present results do not support a key contribution of a defective tubular transport system for phosphate. In the patients with unilateral renal disease, TRP values were identical in the diseased and contralateral kidneys; this observation has previously been made in dogs with unilateral renal disease (1). Moreover, with restoration of plasma calcium concentrations to normal in uremic patients with advanced bilateral renal disease and very low control TRP values, TRP rose to as high as $97.6 \%$, and net reabsorption of phosphate increased, though the filtered load of phosphate decreased by over $70 \%$.

There also is little evidence to support the view that an increase in the filtered load of phosphate plays a major role in the modulation of phosphate excretion. Perhaps the main point against this thesis is that phosphate balance is maintained for a prolonged period of time in chronic renal disease in the absence of hyperphosphatemia. Indeed, the fall in TRP is presumably instrumental in delaying the onset of hyperphosphatemia. It is of interest in this context that lowering phosphate concentrations to below normal levels in the uremic hyperphosphatemic subjects resulted in only a modest increase in TRP values. Furthermore, hemodialysis which lowered GFR as well as plasma phosphate concentrations was attended by a very small rise in TRP values. Therefore, hyperfiltra- 
tion may not be a major factor in modulating phosphate excretion either. The possibility also must be considered that the increase in fractional phosphate reabsorption was due to a direct tubular effect of vitamin $\mathrm{D}$. Two considerations, however, make this explanation unlikely. (a) We are aware of no evidence that vitamin $D$ enhances phosphate reabsorption unless there is an associated increase in serum calcium and a presumed decrease in parathyroid hormone release. (b) In the present studies, TRP did not increase in association with vitamin $\mathrm{D}$ administration until plasma calcium concentrations were restored to normal.

We believe that the data are best explained by invoking a key role of parathyroid hormone in the control of phosphate excretion in advancing renal disease. PTH levels are known to be increased in uremia $(6,7)$. When plasma calcium concentrations were raised to a normal range and presumably PTH excretion was suppressed as a consequence, TRP levels rose strikingly..$^{3}$ This increase occurred at subnormal plasma phosphate concentrations, but it persisted when plasma phosphate concentrations were increased to normal levels. The fact that it required up to 2-3 wk of normocalcemia to reverse the depression of TRP may explain why previous efforts to accomplish this reversal with short-term calcium infusions in uremic subjects have not proven universally successful (8-10).

If parathyroid hormone does play a principal role in the progressive modification of the phosphate excretory profile as renal disease advances, a

\footnotetext{
3 During the course of these studies four uremic patients were encountered in whom TRP values did not increase appreciably in response to correction of hypocalcemia for up to 2 months. In each there was evidence of severe bone disease. Subtotal parathyroidectomy was performed in all. In three of the patients, TRP levels increased to over $80 \%$ postoperatively after vitamin D and calcium therapy. In the fourth patient, TRP remained low, but only two glands were found at surgery, and there is evidence of continuing osteitis fibrosa in this individual. These patients will be described in detail separately.

Two of the patients who responded initially to vitamin $\mathrm{D}$ and calcium also had severe bone disease. In one subtotal parathyroidectomy was performed; in the other, the parathyroid glands were examined at autopsy. There were no histologic features that differentiated the glands of the responsive from the nonresponsive patients.
}

number of questions remain to be answered. Chief among these is the nature of the afferent limb of the control system. If, as seems likely, plasma phosphate concentrations per se are not monitored directly by a sensing mechanism (11), there must be a precise physical feedback between an increase in plasma phosphate concentrations and the parameter that is monitored, most likely some function of serum calcium concentration. If a fall in serum calcium (either total or ionized) does provide the signal for changing rates of $\mathrm{PTH}$ secretion in advancing renal disease, the control system presumably would operate in the following manner. If the intake of phosphate remains relatively constant with time, it may be assumed that the destruction of even the first group of nephrons will lead to positive phosphate balance and to an increase, albeit a minute one, in plasma phosphate concentration. This rise in phosphate concentration would then lead to a reciprocal decrease in calcium concentration. PTH release would increase, TRP would fall, phosphate excretion would rise, plasma phosphate concentration would decrease to the initial level, and the calcium concentration then would return to normal. But the PTH secretory rate would be maintained at a higher than normal level. With each phase of nephron destruction there would be a repetition of the same sequence of events, and PTH levels would rise progressively as the nephron population falls. Although this may be the way that the system does in fact operate, there are difficulties, both conceptual and practical, with such a model. For example, factors other than plasma phosphate concentration must exercise an influence on calcium concentrations in advancing renal disease. Among these are calcium absorption (which in turn will reflect vitamin D responsiveness) (12-16), magnesium concentrations (17), arterial $\mathrm{pH}$ and bone buffering of hydrogen ions (18-20), and possibly thyrocalcitonin levels (21). If one of these factors initiated a change in calcium concentration and thus altered PTH levels independent of any primary change in phosphate balance, or if phosphate transport were altered directly by factors other than PTH, the specificity of the phosphate control system could be impaired. Yet the regulation of plasma phosphate concentrations in advancing renal disease in man seems to be quite precise until GFR values fall below $25 \mathrm{ml} / \mathrm{min}$. A conceptual problem re- 
garding assigning a primary role in the maintenance of phosphate homeostasis to parathyroid hormone is that the key biologic role of PTH presumably relates to the control of bone and calcium metabolism. If in advancing renal disease transient increases in phosphate concentrations occurring during periods of nephron destruction indirectly determine the rate of PTH secretion (via secondary changes in calcium concentrations) the economy of bones would become subservient to the excretion of phosphate by the kidney. A final question relates to the reasons for the delay in the restoration of TRP levels to normal after the induction of normocalcemia. If the biologic half-life of parathyroid hormone is normal in uremia it is conceivable that many patients with far advanced renal disease and hyperparathyroidism of long duration have some degree of autonomy of parathyroid function, and that suppression of hormone release requires sustained correction of hypocalcemia.

It seems quite likely that the solution of these problems will require serial longitudinal measurements of the patterns of renal excretion of phosphate together with measurements of PTH activity, calcium and phosphate balance and plasma concentrations, and possibly other parameters such as magnesium balance and thyrocalcitonin levels as the nephron population is decreased in successive steps in regulated manner in the experimental animal.

\section{ACKNOWLEDGMENTS}

This work was supported by U. S. Public Health Service Research Grant AM-09976, U. S. Graduate Training Grant T1-AM 05248, and Grant FR-36, General Clinical Research Centers Branch, Division of Research Facilities and Resources. Dr. N. S. Bricker is a recipient of a U. S. Public Health Service Career Research Award.

\section{REFERENCES}

1. Slatopolsky, E., L. Gradowska, C. Kashemsant, R. Keltner, C. Manley, and N. S. Bricker. 1966. The control of phosphate excretion in uremia. J. Clin. Invest. 45: 672.

2. Roe, J. H., J. H. Epstein, and N. P. Goldstein. 1949. A photometric method for the determination of inulin in plasma and urine. J. Biol. Chem. 178: 839.

3. Gomori, G. A. 1942. Modification of colorimetric phosphorus determination for use with photoelectric colorimeter. J. Lab. Clin. Med. 27: 955.
4. Slatopolsky, E., I. Elkan, C. Weerts, and N. S. Bricker. 1968. Studies on the characteristics of the control system governing sodium excretion in uremic man. J. Clin. Invest. $47: 521$.

5. Goldman, R., and S. H. Bassett. 1954. Phosphorus excretion in renal failure. J. Clin. Invest. 33: 1623.

6. Berson, S. A., and R. S. Yalow. 1967. Radioimmunoassay of peptide hormones in plasma. New Engl. J. Med. 277: 640.

7. Reiss, E., and J. Canterbury. 1967. A radioimmunoassay for circulating parathyroid hormone in man: preliminary results. J. Lab. Clin. Med. 70: 1012. (Abstr.)

8. Morrin, P. A. F. 1965. Phosphate excretion in chronic uremia with observations on the effect of intravenous infusion of calcium. Metab. Clin. Exptl. 14: 674.

9. Falls, W. F., Jr., N. W. Carter, F. C. Rector, Jr., and D. W. Seldin. 1966. The mechanism of impaired phosphate reabsorption in chronic renal disease. Clin. Res. 9: 74.

10. Better, O., H. C. Gonick, L. Chapman, and C. R. Kleeman. 1965. Renal handling of divalent ions in uremia. Clin. Res. 8: 301.

11. Auerbach, G. D., and J. T. Potts. 1967. Parathyroid hormone. Am. J. Med. 42: 1.

12. I.iu, S. H., and H. I. Chu. 1942. Studies of calcium and phosphorus metabolism with special reference to pathogenesis and effect of dihydrotachysterol (T.A.10) and iron. Medicine. 22: 103.

13. Stanbury, S. W. 1957. Azotaemic renal osteodystrophy. Brit. Med. Bull. 13: 57.

14. Dent, C. E., C. M. Harper, and G. R. Philpot. 1961. The treatment of renal-glomerular osteodystrophy. Quart.J. Med. 30: 1.

15. Fletcher, R. F., J. H. Jones, and D. B. Morgan. 1963. Bone disease in chronic renal failure. Quart. J. Med. 32: 321 .

16. Avioli, L. V., and E. Slatopolsky. 1967. An explanation for the apparent Vitamin D "resistance" in chronic renal disease. J. Lab. Clin. Med. 70: 882. (Abstr.)

17. Gitelman, H. J., S. Kukolj, and L. G. Welt. 1968. The influence of the parathyroid glands on the hypercalcemia of experimental magnesium depletion in the rat. J. Clin. Invest. 47: 118.

18. Pellegrino, E. D., and R. M. Blitz. 1965. The composition of human bone in uremia. Medicine. 44: 397.

19. Goodman, A. D., J. Lemann, Jr., E. J. Lennon, and A. S. Relman. 1965. Production, excretion and net balance of fixed acid in patients with renal acidosis. J. Clin. Invest. $44: 495$.

20. Lemann, J., Jr., J. R. Litzow, and E. J. Lennon. 1966. The effects of chronic acid loads in normal man: further evidence for the participation of bone mineral in the defense against chronic metabolic acidosis. J. Clin. Invest. 45: 1608.

21. Copp, D. H. 1967. Hormonal control of hypercalcemia. Historic development of the calcitonin concept. Am. J. Med. 43: 648. 